\title{
EFECTO AGUDO DEL YOGA Y DE LA DANZA AERÓBICA SOBRE EL ESTADO DE ANIMO Y EL FUNCIONAMIENTO COGNITIVO EN HOMBRES Y MUJERES
}

\author{
Johnny Alberto Montoya Arroyo, Gerardo Araya Vargas y Walter Salazar Rojas \\ Escuela de Educación Física y Deportes \\ Universidad de Costa Rica, San José, Costa Rica \\ E-mail: johnny6@costarricense.cr
}

\section{Resumen}

Montoya-Arroyo, J.A.; Araya-Vargas, G. y Salazar-Rojas, W. (2007). Efecto agudo del yoga y la danza aeróbica sobre el estado de ánimo y el funcionamiento cognitivo en hombres y mujeres. Revista de Ciencias del Ejercicio y la Salud, 5(1), 41-46. El objetivo de este estudio fue establecer una comparación entre un ejercicio aeróbico, como lo es la danza aeróbica, y una actividad de relajación y meditación como lo es el yoga, en cuanto a sus efectos en el estado anímico y en el funcionamiento cognitivo (atención). Se contó con

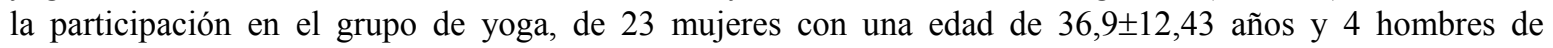

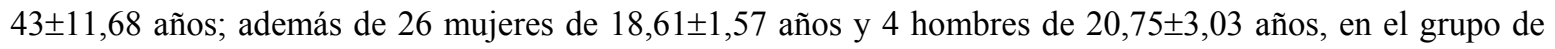
danza aeróbica. Se aplicó la versión de 15 ítems del POMS (medida de estados de ánimo) y el ToulousePieron (medida de atención) antes y después de una sesión de actividad (yoga o danza aeróbica). No se encontró efectos significativos de ninguna de las actividades sobre la atención. En los estados de ánimo, se observó efecto agudo positivo de ambas actividades. En síntesis, la práctica de yoga muestra efectos agudos positivos en el estado de ánimo, similares a los que se obtienen al practicar actividades de tipo aeróbico, como la danza aeróbica. Futuros estudios deben profundizar en los mecanismos psicofisiológicos implicados en estos cambios.

Palabras clave: aeróbicos, yoga, estado de ánimo, concentración y ejercicio.

\section{INTRODUCCION}

Los resultados que se han obtenido en el campo de la actividad física y el ejercicio, y el impacto que tiene sobre la salud de los seres humanos, revelan una serie de beneficios, que brinda el llevar a cabo con regularidad la práctica de alguna de estas actividades, los mismos van desde mejoras en el estado y funcionamiento físico, hasta ser un medio para combatir problemas como el estrés, ansiedad y otros problemas psicológicos (Dishman y Jackson, 2000; Courneya y Hayduk, 2002; Petruzzello, 1995).

Pero no solo se ha estudiado el efecto de la práctica regular, sino que también se han realizado estudios como los de Petruzzello, Landers, Hatfield, Kubitz y Salazar (1991); Berger y Owen (1998); Netz y Lidor (2003); en los cuales se ha buscado explicar el efecto inmediato de una sola sesión de ejercicio, y éstos han comprobado su impacto agudo a nivel psíquico y emocional de las personas.
Así mismo el efecto inmediato o agudo de una sesión de ejercicio ha sido comprobado utilizando diferentes ejercicios de tipo aeróbico como lo son: carrera y natación (Berger y Owen, 1998, 1992), ciclismo (Reed, Berger, Latin y LaVoie, 1998; Koltyn, Lynch y Hill, 1998), danza aeróbica (Kennedy y Newton, 1997) y ejercicios en el agua (Oda, Matsumoto, Nakagawa y Moriya, 1999); sin embargo de acuerdo con los resultados obtenidos en el meta-análisis de Petruzzello y cols. (1991), no se presentan diferencias significativas entre varios tipos de ejercicios aeróbicos, así como tampoco se ha encontrado que una actividad sea mejor que las demás, tomando el tamaño del efecto de diferentes ejercicios de tipo aeróbico.

Sin embargo, se han realizado muy pocos estudios en donde se compara el efecto agudo del ejercicio aeróbico y otras modalidades, como los ejercicios en los que se requiere de meditación y relajación, como el tai chi, yoga, Feldenkrais y otros ejercicios derivados, los cuales combinan la meditación 
con movimientos suaves y acciones musculares estáticas, como los realizados por Berger y Owen (1992); y Netz y Lidor (2003). Este tipo de actividades han sido tomadas en cuenta principalmente en estudios con características clínicas, por los diferentes beneficios que se le atribuyen a la meditación (Delmonte, 1984), además es en el yoga en el que se ha documentado más frecuentemente investigaciones de esta naturaleza.

Es así, que el objetivo de este estudio, es el establecer una comparación entre un ejercicio aeróbico, como lo es la danza aeróbica, y una actividad de relajación y meditación como lo es el yoga. Se debe tener claro en qué consisten tanto la danza aeróbica como el yoga; en referencia a la danza aeróbica, desde hace más de 20 años se inició como una forma de trabajo para mejorar la condición física, cuando Kenneth Cooper, específicamente en 1968, publicó su libro "Aerobics", el cual presentaba una serie de ejercicios para mejorar el sistema cardiaco y pulmonar. La estructura de éste consistía en 10 a $15 \mathrm{~min}$. de calentamiento, de 6 a $10 \mathrm{~min}$. de danza aeróbica y al final un trabajo de danza y rutina de estiramiento.

Sin embargo, en la actualidad se ha modificado un poco esta estructura, pues una clase puede constar de 10min. de calentamiento, de 20 a $30 \mathrm{~min}$. de trabajo aeróbico, que puede ser de baja o alta intensidad (se define por los pasos que se utilizan) de acuerdo al nivel de los participantes, luego una normalización de 5 min., un trabajo de piso y/o localizado que va de 10 a $15 \mathrm{~min}$., y un estiramiento de $5 \mathrm{~min}$. para finalizar.

Con respecto al yoga, esta es una de las seis escuelas filosóficas de la india, la cual se basa en promover la búsqueda de un balance entre el cuerpo, el espíritu, la mente y el aspecto social, y también es utilizada como parte del sistema médico tradicional de la India; sus orígenes se remontan aproximadamente alrededor del año 3000 antes de Cristo, como prueba de ello, en lugares como los Valles del Indo y Saraswati, se han hallado sellos de piedra con figuras de posiciones yóguicas, así mismo la escrituras hindúes se encuentran entre las más antiguas del mundo y en ellas se hace referencia al yoga. El yoga contemporáneo inicia hacia 1900, donde diferentes maestros llegaron a occidente creándose así el origen de las distintas escuelas conocidas actualmente (Feuerstein, 1998; Lamb, 2001; Cowen y Adams, 2004).

El ashtanga yoga, o yoga de los ocho pasos incluye un conjunto de técnicas complementarias entre sí, que constituyen toda la práctica del yogui, los cuales son: 1) Yama: actitudes éticas (no violencia, veracidad, honestidad, moderación y posesión justa); 2) Niyama: observancias (pureza física y mental, contento, disciplina, estudio y desapego); 3) Asana: posiciones físicas; 4) Pranayama: control de la respiración; 5) Pratyahara: abstracción sensorial; 6) Dharana: concentración; 7) Dyana: meditación; y 8) Samadhi: interiorización profunda; sin embargo estos pasos no se consideran como un proceso lineal (Ruiz, 2001; Cowen y Adams, 2004; The ethical foundation of yoga, 2004).

A la práctica del yoga, se le atribuyen una serie de beneficios tanto físicos como psíquicos, a causa de sus posturas, la meditación y relajación que son parte del desarrollo de esta actividad; dentro de los beneficios psicológicos están: ayuda a reducir los niveles de estrés, disminuye el riesgo de padecer o presentar recaídas de problemas de depresión mayor, presenta una significativa reducción de la depresión y la ansiedad, incrementa el auto-eficacia, mejora la autoaceptación, la concentración, la memoria y la atención, e incrementa el nivel de energía en las personas; beneficios físicos: ayuda a reducir dolores crónicos, el ejercicio de respiración (Pranayama) mejora la función inmunológica, disminuye el pico de presión respiratorio durante la ventilación por lo que incrementa la percepción de la ventilación durante la inspiración, promueve la fuerza y la resistencia muscular, mejora la flexibilidad y la elasticidad (según Arpita, 1990; Lamb, 2001; Malathi, Neela, Nilesh,, Damodaran y Marathe, 2001; Weelde y Thompson, 2004; Kamei, Toriumi, Kimura y Kimura, 2001; Monnazzi, Leri, Guizzardi, Mattioii y Patacioli, 2002; The ethical foundation of 
yoga, 2004; Villien, Yu, Barthélémy, y Jammes, 2004; Cowen y Adams, 2004).

El funcionamiento cognitivo y el estado de ánimo, son indicadores importantes de la calidad de vida de las personas, por lo cual han sido motivo de estudio en las ciencias del movimiento humano y la salud (Brannon y Feist, 2001; Gutiérrez, Raich, Sánchez y Deus, 2003). Se han demostrado los efectos positivos del ejercicio aeróbico, pero queda por conocerse aún si otras actividades con intensidades de esfuerzo más bajas, como el yoga, podrían tener beneficios similares, lo cual indicaría que el mecanismo fisiológico del ejercicio aeróbico no sería suficiente para explicar los cambios observados en esas variables. De aquí parte la relevancia del estudio.

\section{METODOLOGIA}

Sujetos: se contó con la participación en el grupo de yoga, de 23 mujeres de $36,9 \pm 12,43$ años y 4 hombres con un promedio de edad

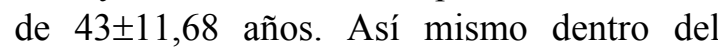
grupo de danza aeróbica se contó con la participación de 26 mujeres de 18,61 $\pm 1,57$ años y 4 hombres de 20,75 3 3,03 años.

Instrumentos: se utilizó una adaptación española del Profile of Mood States, (POMS), de McNair, Lorr y Droppleman (1971), por Balaguer y cols. (1993), "Perfil de Estados de Ánimo", el cual posee 15 ítems, y evalúa cinco estados de ánimo: tensión, depresión, cólera, vigor y fatiga y el índice global del estado de ánimo negativo, el cual se calcula sumando los puntajes de tensión, depresión, cólera y fatiga y restándole el puntaje de vigor y sumándole una constante de valor 12 . También se utilizó la prueba pictográfica de Toulouse-Pieron, para la evaluación de aptitudes perceptivas y de atención. Ambos instrumentos cuentan con reportes de validez y se han aplicado exitosamente en el contexto de la psicología del deporte y el ejercicio.

Procedimiento: se asistió a diferentes centros en donde las personas se reúnen para practicar las disciplinas elegidas, como los estudios de yoga y gimnasios. Se solicitaron los permisos requeridos en los diferentes centros así como a cada una de las personas; una vez que accedían a participar, llenaron una hoja de consentimiento. La administración de las pruebas se llevó a cabo en dos sesiones diferentes, el POMS requirió aproximadamente de 5 minutos para su administración, mientras que el test de Toulouse-Pieron, requería 8 minutos. Ambas pruebas se aplicaron exactamente antes de iniciar la sesión ya fuese de yoga o de aeróbicos, y volvieron a ser aplicadas exactamente después de terminada la sesión, para poder establecer de esta manera el efecto agudo de la actividad en los participantes.

Análisis estadístico: como estadística descriptiva se calculó promedios y desviaciones estándar; como estadística inferencial, se aplicó el análisis de correlación de Pearson, el análisis de covarianza (ANCOVA) tomando como covariable la edad, y además el análisis de varianza (ANOVA) de medidas repetidas. Todos los cálculos se realizaron con el programa estadístico SPSS versión 8.0 para Windows.

\section{RESULTADOS}

El análisis de correlación de Pearson mostró que la edad de los sujetos se correlacionaba significativamente con las mediciones pre y post de atención, por lo que se concluyó que esa variable podría afectar los resultados y por tanto era necesario controlarla. Por esto, se realizaron análisis de covarianza tomando como covariable a la edad, para removerla estadísticamente en el caso de la variable de atención.

\begin{tabular}{|c|c|c|c|c|}
\hline & & Promedio* & Error estándar & $\mathrm{n}$ \\
\hline \multirow[t]{3}{*}{ Pre } & Aeróbicos & 81,4333 & 16,2601 & 30 \\
\hline & Yoga & 82,1111 & 16,7087 & 27 \\
\hline & Total & 81,7544 & 16,3295 & 57 \\
\hline \multirow[t]{3}{*}{ Post } & Aeróbicos & 88,1667 & 11,6177 & 30 \\
\hline & Yoga & 86,6667 & 15,1734 & 27 \\
\hline & Total & 87,4561 & 13,3176 & 57 \\
\hline
\end{tabular}


*Ajustados según la edad como covariable (edad=27,8596)

En los resultados de la prueba de ToulousePieron, se marca una tendencia a la mejora en ambas disciplinas, sin embargo las diferencias no fueron estadísticamente significativas (mediciones $\mathrm{F}=0,175$ y mediciones $\mathrm{X}$ actividad $\mathrm{F}=0,441$; ambas $\mathrm{p}>0,05$ ).

En cuanto a los estados de ánimo (ver tabla 2), en tensión-ansiedad se encontró diferencias significativas entre mediciones, pero tampoco hubo interacción entre grupos y mediciones, por tanto la tendencia es similar para ambos tipos de actividad; en general se observa un aumento significativo en la tensión (mediciones $\mathrm{F}=19,951 ; \mathrm{p}<0,01$; actividad $\mathrm{F}=6,506 ; \mathrm{p}>0,05$ y mediciones $\mathrm{X}$ actividad $\mathrm{F}=0,035 ; \mathrm{p}>0,05$ ).

En lo que se refiere a los puntajes de depresión, se encontraron diferencias 43 estadísticamente significativas entre mediciones y actividades, sin embargo tampoco hubo interacción significativa; ambas actividades presentan una mejoría similar en el nivel de depresión aguda (mediciones $\mathrm{F}=12,37 ; \mathrm{p}<0,01 ; \quad$ actividad $\mathrm{F}=1,987 ; \mathrm{p}>0,05$ y mediciones $\mathrm{X}$ actividad $\mathrm{F}=0,693 ; \mathrm{p}>0,05)$.

Tabla 2. Resumen de promedios y desviación estándar, de los puntajes de estados de ánimo, en practicantes de yoga y danza aeróbica

\begin{tabular}{|c|c|c|c|c|c|c|}
\hline & \multicolumn{2}{|c|}{ Aeróbicos (n: 30) } & \multicolumn{2}{|c|}{ Yoga (n: 27) } & \multicolumn{2}{|c|}{ Total (n: 57) } \\
\hline & Pre & Post & Pre & Post & Pre & Post \\
\hline Tensión & $19,3 \pm 2,6$ & $20,9 \pm 2,5$ & $20,7 \pm 2,8$ & $22,4 \pm 2,0$ & $19,9 \pm 2,7$ & $21,6 \pm 2,4$ \\
\hline Depresión & $13,1 \pm 1,4$ & $12,5 \pm 1,1$ & $12,5 \pm 1,6$ & $12,2 \pm 0,8$ & $12,8 \pm 1,5$ & $12,3 \pm 0,9$ \\
\hline Cólera & $17,0 \pm 2,8$ & $16,6 \pm 2,5$ & $15,0 \pm 2,5$ & $13,5 \pm 1,9$ & $16,1 \pm 2,8$ & $15,1 \pm 2,7$ \\
\hline Vigor & $17,1 \pm 2,5$ & $19,0 \pm 3,1$ & $19,6 \pm 2,7$ & $21,2 \pm 2,9$ & $18,3 \pm 2,9$ & $20,1 \pm 3,2$ \\
\hline Fatiga & $13,0 \pm 1,8$ & $12,4 \pm 0,8$ & $12,7 \pm 1,9$ & $12,2 \pm 0,9$ & $12,8 \pm 1,8$ & $12,3 \pm 0,9$ \\
\hline
\end{tabular}

Tabla 3. Resumen de análisis de promedios y desviaciones estándar de los puntajes del índice global de estado de ánimo negativo según tipo de actividad y medición

\begin{tabular}{|c|c|c|c|c|}
\hline \multicolumn{3}{|c|}{ Actividad } & & \multirow{2}{*}{$\frac{\mathrm{n}}{30}$} \\
\hline pre test & Aeróbicos & 45,4667 & $\pm 5,9639$ & \\
\hline & Yoga & 41,4074 & $\pm 6,0464$ & 27 \\
\hline & Total & 43,5439 & $\pm 6,2908$ & 57 \\
\hline \multirow[t]{3}{*}{ post test } & Aeróbicos & 43,8000 & $\pm 5,1152$ & 30 \\
\hline & Yoga & 39,1481 & $\pm 4,3119$ & 27 \\
\hline & Total & 41,5965 & $\pm 5,2606$ & 57 \\
\hline
\end{tabular}


En cuanto a los puntajes de cólera, también se encontraron diferencias estadísticamente significativas entre mediciones y entre actividades, pero tampoco se dio interacción significativa. Por tanto, en ambas actividades se dio disminución aguda de la cólera (mediciones $\mathrm{F}=6,704 ; \mathrm{p}<0,05 ;$ actividad $\mathrm{F}=22,831 ; \mathrm{p}<0,01$ y mediciones $\mathrm{X}$ actividad $\mathrm{F}=2,341 ; \mathrm{p}>0,05)$.

En los puntajes de vigor se presentan diferencias estadísticamente significativas entre las mediciones y actividades, sin embargo tampoco no se dio interacción significativa. Por tanto, en ambas actividades se encontró un aumento agudo del vigor (mediciones $\mathrm{F}=18,1 ; \mathrm{p}<0,01 ;$ actividad $\mathrm{F}=15,369 ; \mathrm{p}<0,01$ y mediciones $\mathrm{X}$ actividad $\mathrm{F}=0,132 ; \mathrm{p}>0,05)$.

Con respecto a la fatiga, se presentaron diferencias estadísticamente significativas entre mediciones, pero no así entre actividades y tampoco hubo interacción significativa. Por tanto, en ambas actividades disminuyó el nivel de fatiga (mediciones $\mathrm{F}=7,699 ; \mathrm{p}<0,05 ;$ actividad $\mathrm{F}=0,594 ; \mathrm{p}>0,05$ $y$ mediciones $X$ actividad $F=0,308 ; p>0,05$ ). En cuanto al índice global de estado de ánimo negativo (ver tabla 3), se encontraron diferencias estadísticamente significativas entre mediciones y entre actividades, pero tampoco se presentó interacción significativa, en esta variable (mediciones $\mathrm{F}=5,952$; $\mathrm{p}<0,05 ;$ actividad $\mathrm{F}=13,405 ; \quad \mathrm{p}<0,01 \quad y$ mediciones $\mathrm{X}$ actividad $\mathrm{F}=0,136 ; \mathrm{p}>0,05)$. Por tanto, existe una mejora aguda significativa del nivel de estado de ánimo negativo luego de realizar aeróbicos o la sesión de yoga.

\section{DISCUSIÓN}

El propósito de este estudio fue comparar los beneficios a nivel agudo, que podrían brindar dos actividades de características tan distintas, como lo son la danza aeróbica y el yoga, sobre el estado de ánimo y la cognición. Los resultados obtenidos son consistentes con los de Berger y Owen (1992), y de Netz y Lidor (2003), ya que al establecer una comparación entre las puntuaciones obtenidas en el estado de ánimo y la concentración, de un grupo de practicantes de danza aeróbica y otro grupo de practicantes de yoga, no se presentaron diferencias estadísticamente significativas entre las actividades.

En cuanto a los resultados del estado de ánimo, se mostraron beneficios agudos de la práctica de la danza aeróbica (según se esperaba de acuerdo a la evidencia previa como por ejemplo en Petruzzello, 1995 y Petruzzello y cols., 1991) y al practicar una sesión de yoga. Varios estudios han demostrado la efectividad del yoga para mejorar los estados anímicos (Arpita, 1990; Berger y Owen, 1992; Cowen y Adams, 2004). Sin embargo, es necesario profundizar esta línea de investigación, sobre todo para entender mejor los mecanismos psicofisiológicos que intervienen en los beneficios reportados.

Además, con respecto al índice global de estado de ánimo negativo, se encontraron diferencias significativas entre mediciones y actividades, pero al igual que en las otras variables dependientes no se mostró interacción significativa. En ambas actividades (danza aeróbica y yoga) las tendencias tuvieron un mismo comportamiento, presentándose mejoras en el índice global del estado ánimo, el cual es un mejor indicador de la variación del estado de ánimo negativo para la versión del POMS que fue utilizada (Fernández-Castro y BlascoBlasco, 2003). En síntesis, la práctica de yoga muestra efectos agudos positivos en el estado de ánimo, similares a los que se obtienen al practicar actividades de tipo aeróbico, como la danza aeróbica, no obstante no se podría concluir que los mecanismos psicofisiológicos responsables de esos cambios sean los mismos, lo cual debe analizarse a profundidad en futuros estudios. Pero resulta claro que existe evidencia de un mecanismo común en actividades aeróbicas y de meditación como el yoga, que podría explicar los efectos agudos en el estado 
anímico. Se debe profundizar más estos hallazgos.

\section{REFERENCIAS}

Arpita. (1990) Physiological and psychological effects of Hatha yoga: A review of the literature. The Journal of The International Association of Yoga Therapists, 1(I\&II):1-2

Berger, B. G. \& Owen, D.R. (1992) Mood alteration with yoga and swimming: aerobic exercise may not be necessary. Perceptual and Motor Skills.75: 1331-1343.

Berger, B. G. \& Owen, D.R. (1998) Relation of low and moderate intensity exercise with acute mood change in college joggers. Perceptual and Motor Skills.87: 611-621.

Brannon, L. y Feist, J. (2001). Psicología de la salud. Madrid, España: Paraninfo - Thomson learning.

Cowen, V.S. \& Adams, T.B. (2004) Physical and perceptual benefits of yoga asana practice: results of a pilot study. Journal of Bodywork and Movement Therapies.

Delmonte, M. (1984) Physiological concomitants of meditation practice. International Journal of Psychosomatics, 31(4):23-36.

Dishman, R.K. \& Jackson, E. (2000) Exercise, Fitness, and Stress. International Journal Psychology. 31: 175-203.

Fernández-Castro, J. y Blasco-Blasco, T. (2003). Instrumentos para la valoración del estrés. En: Gutiérrez, T.; Raich, R.M.; Sánchez, D. y Deus, J. (coords.) Instrumentos de evaluación en psicología de la salud. Madrid, España: Alianza editorial.

Feuerstein, G. (1998) Yoga and Yoga Therapy, International Association of Yoga Therapists.

Gutiérrez, T.; Raich, R.M.; Sánchez, D. y Deus, J. (coords.) (2003). Instrumentos de evaluación en psicología de la salud. Madrid, España: Alianza editorial.

Kennedy, M. \& Newton, M. (1997) Effect of exercise intensity on mood in step aerobics. Journal of Sports Medicine and Physical Fitness. 37: 200-204.

Kamei, T., Toriumi, Y., Kimura, H. \& Kimura, K. (2001) Correlation between alpha rhythms and natural killer cell activity during yogic respiratory exercise. Stress and Health. 17: 141-145.

Koltyn, K., Lynch, N. \& Hill, D. (1998) Psycological responses to brief exhaustive cycling exercise in the morning and the evening. International Journal of Sport Psychology. 29: 145-156.

Lamb, T. (2001) Health Benefits of Yoga. Yoga World. $16, \mathrm{p} 6$.

Malathi, A., Neela, P, Nilesh, S, Damodaran, \& Marathe, M. (2001) Promotive, prophylactic benefits of yogic practices in middle-aged women. International Journal of Yoga Therapy, forthcoming, no. 11

Monnazzi, P., Leri, O., Guizzardi, L., Mattioii, D. \& Patacioli, F.R. (2002) Anti-stress effect of yoga type breathing: modification of salivary cortisol, heart rate and blood pressure following a step-climbing exercise. Stress Health. 18: 195-200.

Netz, Y. \& Lidor, R. (2003) Mood alterations in mindful versus aerobic exercise modes. Journal of Psychology. 137: 0022-3980.

Oda, S., Matsumoto, T., Nakagawa K. \& Moriya, K. (1999) Relaxation effects in humans of underwater exercise of moderate intensity. European Journal of Applied Physiology. 80: 253-259.

Petruzzello, S.J. (1995) Anxiety reduction following exercise: methodological artifact or "real" phenomenon?. Journal of Sport and Exercise Psychology. 17: 105-111.

Petruzzello, S.J., Landers, D.M., Hatfield, B.D., Kubitz, K.A \& Salazar, W. (1991) A meta-analysis on the anxiety reducing effects of acute and chronic exercise. Sport Medicine. 11: 143182.

Programa de curso EF-0113 Electiva Danza Aeróbica, de la Escuela de Educación Física y Deportes, Universidad Costa Rica. (1999).

Reed, J., Berger, K., Latin, R., \& LaVoie, J. (1998) Affective responses of physically active and sedentary individuals during and after moderate aerobic exercise. Journal of Sports Medicine and Physical Fitness. 38: 272-278.

Ruiz, F. (2001) Don't know much philosophy. Yoga Journal. 159: 143-146.

The ethical foundation of yoga. (2004) Legal and ethical issues. Journal of Professional Nursing. Pp. 275-276.

Villien, F., Yu, M., Barthélémy, P. \& Jammes, Y. (2004) Training to yoga respiration selectively increases respiratory sensation in health man. Respiratory Physiology and Neurobiology.

Weelde, L.C. \& Thompson, L. (2004) Apilot study of yoga and meditation intervention for dementia caregiver stress. Journal of Clinical Psychology. 60 (6): 677-687. 